\title{
Feasibility of MRI in diagnosis and characterization of intra-articular synovial masses and mass-like lesions
}

\author{
Nesreen Mohey ${ }^{*}$ (1) and Tamir A. Hassan
}

\begin{abstract}
Background: Classifications of intra-articular masses are infectious granulomatous diseases, non-infectious synovial proliferative processes (synovial chondromatosis, lipoma arborescens, rheumatoid arthritis, pigmented villonodular synovitis [PVNS]), vascular malformations, deposition disorders, neoplastic, and miscellaneous conditions. The aim of this study is to correlate the results of MRI with arthroscopy in characterization of different varieties of intra-articular synovial masses and mass-like lesions. This observational prospective study was conducted between February 2018 and August 2019. We screened 1000 routine musculoskeletal MRI examination during this period, 32 of which showed intra-articular synovial masses/mass-like lesions. The selected 32 patients (20 males and 12 females) have a mean age of $49.20 \pm 2.0$ years; all presented with joint swelling, pain, or difficulty of movement according to the joint affected. All patients underwent arthroscopy for histopathological correlation.

Results: The final diagnosis was synovial chondromatosis in 14 patients (43.8\%), PVNS in ten patients (31.3\%), and lipoma arborescens in five patients (15.6\%). Synovial chondrosarcoma, synovial hematoma, and synovial ganglion cyst each in one patient (3.1\%). The concordance of MRI to arthroscopy was $96.6 \%$ accuracy, $91.7 \%$ sensitivity, $99 \%$ specificity, 52.3\% PPV, and 99.9\% NPV.
\end{abstract}

Conclusion: Good correlation was observed between MRI and arthroscopy in diagnosis of intra-articular synovial masses/mass-like lesions.

Keywords: MRI, Intraarticular synovial masses

\section{Background}

The synovial membrane is originating from embryonic mesenchyme and outline nonarticular areas in synovial joints, sheaths of the tendons, and bursae. Synovial membrane cells produce hyaluronate, which is a main component of the synovial fluid and normalize the exchange of substances between synovial fluid and the blood [1].

Simple classification of intra-articular masses as infectious granulomatous diseases (tuberculous and coccidioidomycosis arthritis), noninfectious synovial proliferative processes (synovial chondromatosis, lipoma arborescens, rheumatoid arthritis, pigmented villonodular synovitis [PVNS]), vascular malformations

\footnotetext{
* Correspondence: nesreenmohey77@yahoo.com

Radiology Department, Faculty of Medicine, Zagazig University, Zagazig, Egypt
}

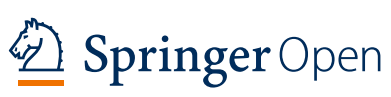

(c) The Author(s). 2020 Open Access This article is distributed under the terms of the Creative Commons Attribution 4.0 International License (http://creativecommons.org/licenses/by/4.0/), which permits unrestricted use, distribution, and reproduction in any medium, provided you give appropriate credit to the original author(s) and the source, provide a link to the Creative Commons license, and indicate if changes were made.

(arteriovenous malformations, synovial hemangioma), deposition disorders (amyloid arthropathy, gout), neoplastic (synovial sarcoma, synovial chondrosarcoma, synovial metastases), and miscellaneous conditions (cyclops lesion following arthroscopic repair of anterior cruciate ligament of the knee) [2,3].

Early imaging is highly important in the early detection of synovial disorders to avoid the incidence of irreversible joint injury. Conventional radiography, joint high-frequency ultrasonography (US), and musculoskeletal magnetic resonance imaging (MRI) are used in the diagnosis of synovial lesions and in follow-up [4].

MRI often done before arthroscopic procedures, and usually management planes are based upon MRI findings [5].

The aim of this study is to correlate the results of MRI with arthroscopy in characterization of different 

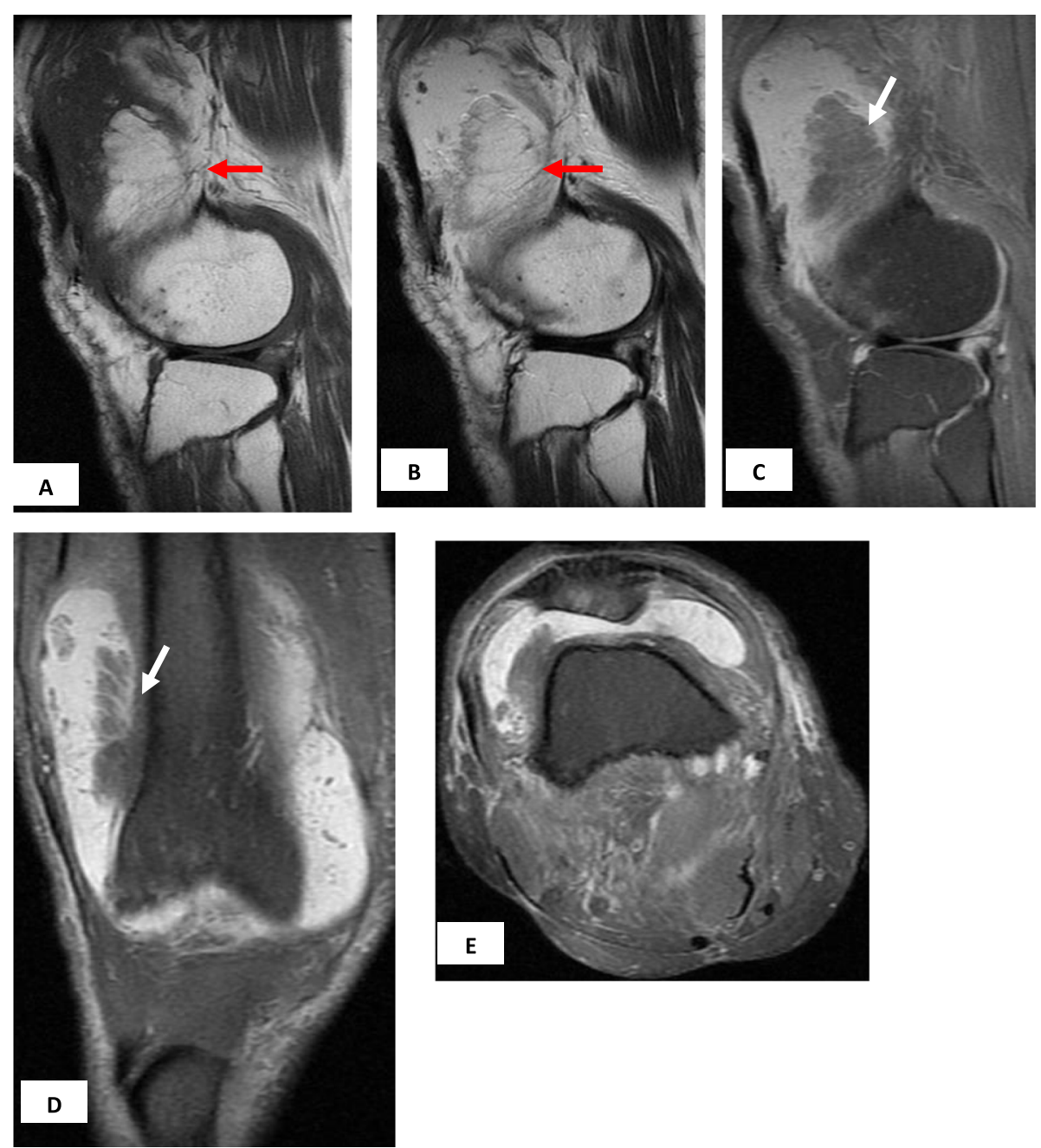

Fig. 1 Lipoma arborescence in 49-year-old male with knee swelling. Sagittal T1 (a) and sagittal T2 (b) show suprapatellar lobulated fat signal lesion (red arrows). Sagittal (c), coronal (d), and axial (e) STIR show loss of signal of the synovial fatty lesion (white arrows)

varieties of intra-articular synovial masses and mass like lesions.

\section{Methods}

\section{Patients}

This prospective study was conducted between February 2018 and August 2019. We screened 1000 routine musculoskeletal MRI examination during this period referred to the MRI unit in our institution, 32 of which met our inclusion criteria and showed intra-articular synovial-based masses or mass-like lesions. The selected 32 patients ( 20 males and 12 females) have a mean age of $49.20 \pm 2.0$ years (range 25-70 years); all presented with joint swelling, pain, or movement limitation according the joint affected. All patients gave their informed consent and this study was approved by the local institutional review board in accordance with the Declaration of Helsinki regarding research involving human subjects.

Inclusion criteria included any intra-articular synovialbased mass or mass-like lesions in adult age groups (18 years and above).

Exclusion criteria included operated cases for synovial-based lesions, juxta-articular synovial lesions, contra-indication to MRI, and patients refusing arthroscopy.

All patients underwent detailed history taking, general and local examination of the affected joint. Laboratory investigation for renal function tests if MRI contrast was decided to be administered.

All the selected 32 cases underwent arthroscopy as a gold standard for histopathological correlation. 

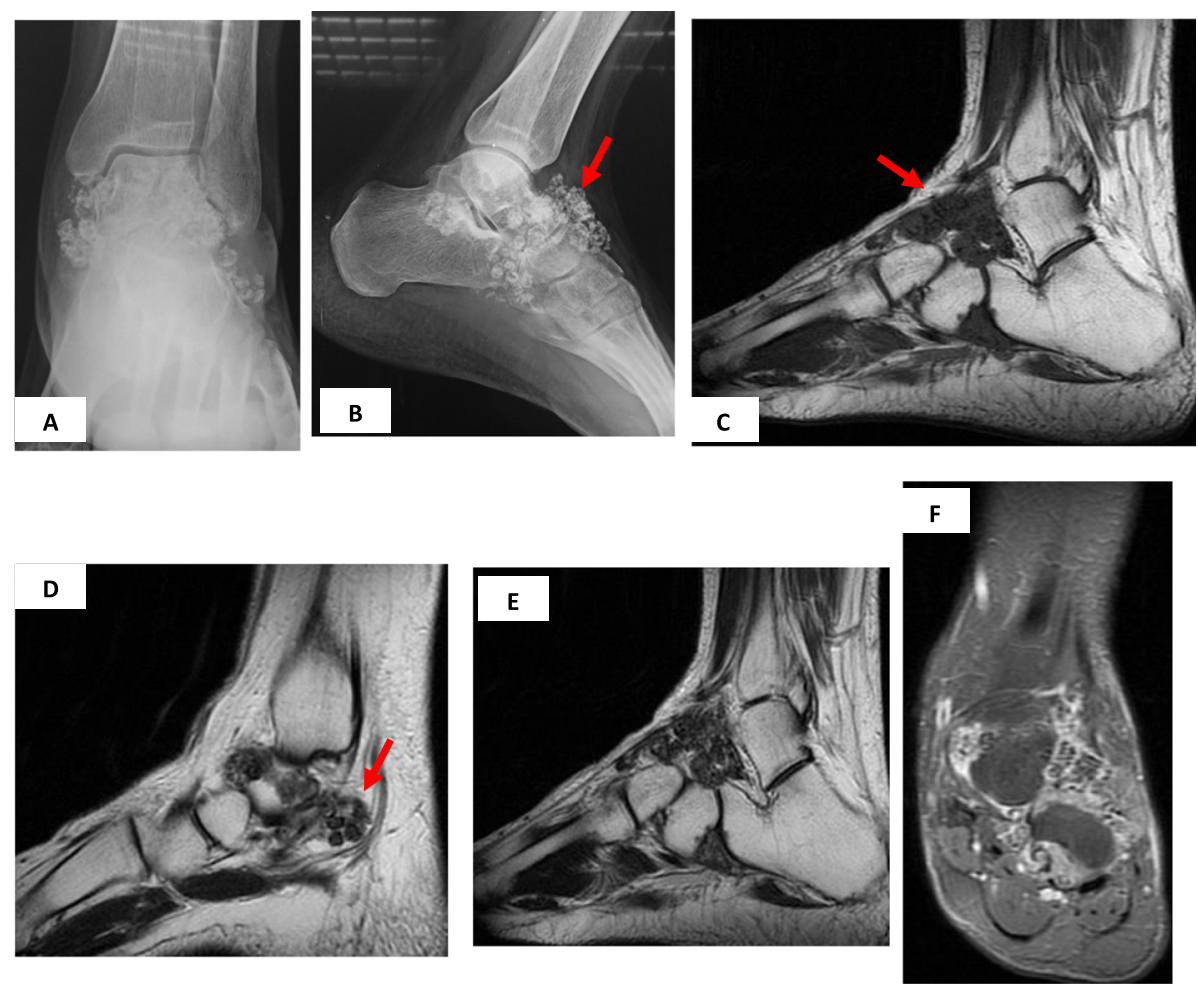

Fig. 2 Synovial chondromatosis in 52-year-old male with left ankle swelling. X-ray AP and Lat views (a, b) show multiple rounded uniform size calcified nodules (red arrows) around the ankle. Sagittal T1 (c), sagittal T2 (d, e), and coronal STIR (f) show low signal at T1 of the calcified nodules, low signal intensity of the periphery of the nodules with high signal of center due to bone marrow fat on T2 and STIR

\section{MRI technique}

All MRI studies for the 32 patients were obtained using a closed MRI machine (General Electric SIGNA) HS (high speed) with magnets of intensity field $1.5 \mathrm{~T}$ and gradients $20 \mathrm{MT} / \mathrm{m}$, equipped with dedicated knee coil (for the knee and the ankle) and abdominal flex coli for the hip joint.

\section{Image acquisition}

Pulse sequences were chosen according to the joint examined with additional sequences used depending on the pathology found during the exam (e.g., gradient sequences GRE in case of suspected PVNS). The routine sequences $\mathrm{T} 1$-weighted fast spin echo (FSE) images (with repetition time (TR) $600 \mathrm{~ms}$ and echo time (TE) $15 \mathrm{~ms}$ ), T2-weighted FSE images (with TR $4000 \mathrm{~ms}$ and TE $90 \mathrm{~ms}$ ), and short T1/Tau (STIR) images (with TR over $4000 \mathrm{~ms}$ and TE $120 \mathrm{~ms}$ ) were used in all joints in different planes. Proton density weighted images were used in the knee joint (with TR over $2000 \mathrm{~ms}$ and TE 10$20 \mathrm{~ms}$ ). T2-weighted images with fat suppression (with TR over $3000 \mathrm{~ms}$ and TE $80 \mathrm{~ms}$ ) when needed. Slice thickness was $4.0 \mathrm{~mm}$ in all sequences.
Inter slice gap $1 \mathrm{~mm}$. Field of view (FOV): 14$16 \mathrm{~cm}$ depending on the size of the patient. Matrix: $256 \times 256$ and the flip angle $=250$.

Intravenous contrast-enhanced sequences were obtained in five cases with PVNS showing diffuse synovial thickening and this was achieved by intravenous injection of magnevist $0.1 \mathrm{mmol} / \mathrm{kg}$ [gadolinium diethylenetriaminepenta-acetic acid (Gd-DTPA); Berlex, Montvale, $\mathrm{NJ}$ ] after making sure that the renal function test was good.

\section{Image interpretation}

Images were transferred to the PACS workstations (Philips, iSite radiology) and independently reviewed by two experienced musculoskeletal radiologists (over 10-years' experience in MRI musculoskeletal imaging). Each radiologist assessed the affected joint for the routine exam then meticulous evaluation of any intraarticular synovial-based lesions regarding its site, size, signal intensity, extension, articular surfaces erosions, and possible diagnosis. In case of controversy between the two observers, this is solved by consensus after conjoint evaluation of the MRI images. 

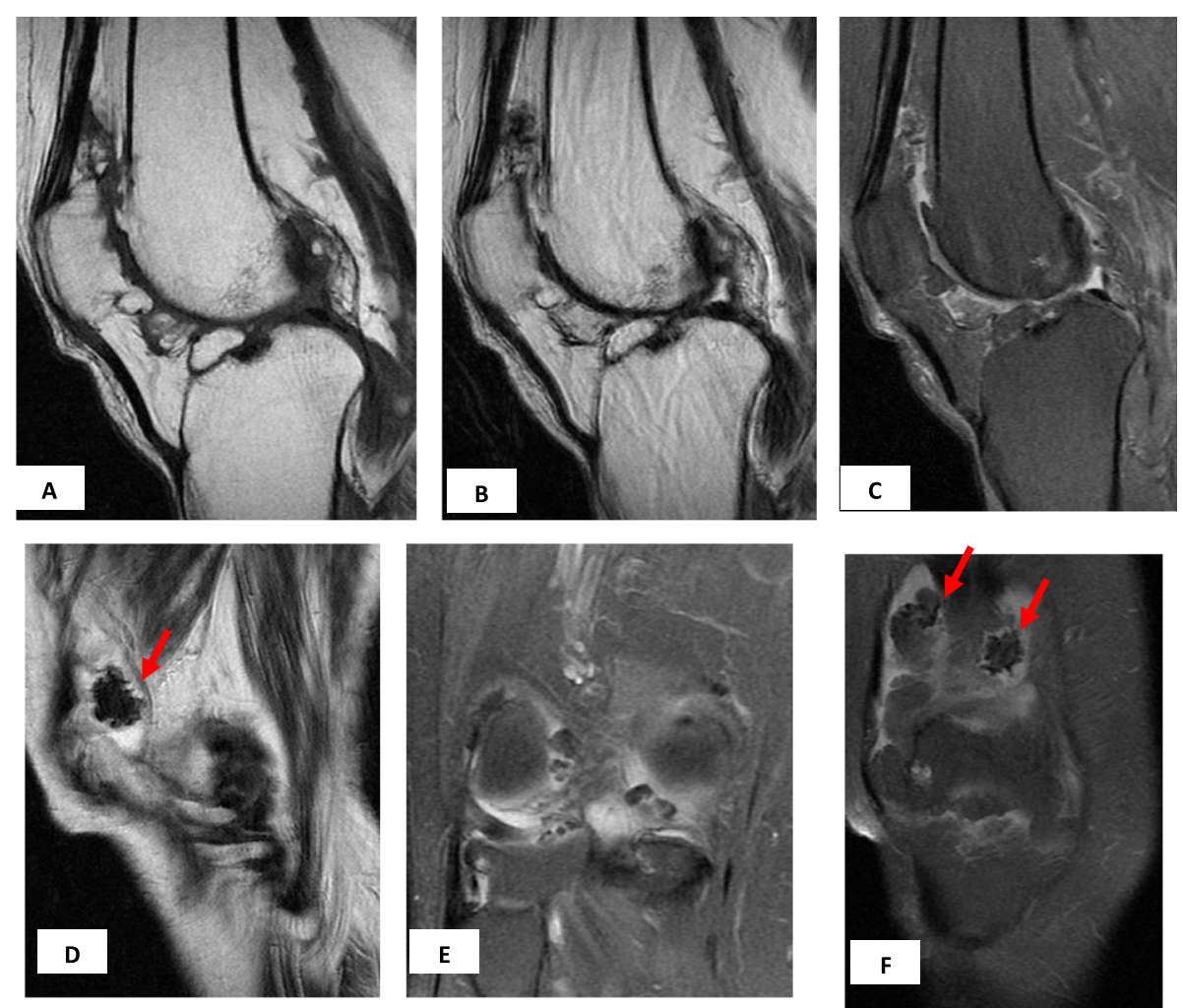

Fig. 3 Synovial chondromatosis in 58-year-old male with right knee osteoarthritis. Sagittal T1 (a), sagittal T2 (b, d), sagittal STIR (c), and coronal STIR (e, f) show low signal at T1, T2, and STIR calcified different size nodules (red arrows) with osteoarthritic changes

\section{Final diagnosis was achieved by}

Arthroscopy was done within 7 days after MRI examination to all selected patients and was performed in the orthopedic department. The results of arthroscopy and histopathology results were later correlated with MRI.

\section{Statistical analysis}

The data were collected, tabulated, and analyzed statistically. All statistical analyses were computed with the Statistical Package for the Social Sciences (SPSS) Version 21. Descriptive statistic was performed in a form of number and percentage for qualitative data. Sensitivity, specificity, and diagnostic accuracy of MRI in evaluation of synovial intra-articular lesions were calculated and correlated with arthroscopy.

The inter-observer agreement for assessing the synovial lesions was calculated. The percentage of agreement as well as the kappa statistic was used to calculate the percent of interpreter agreement above the expected by chance.

Moderate agreement was considered when weighted kappa statistic of 0.41-0.60, good agreement of 0.610.80 , and excellent agreement of $0.81-1.00$ [6].

\section{Results}

This prospective study included 32 selected patients performed routine musculoskeletal MRI according to the study inclusion and exclusion criteria; males $(62.5 \% \%)$ were affected more than females $(37.5 \% \%)$ with mean age of $49.20 \pm 2.0$ years (range 2570 years).

The most affected joint was the knee joint (90.6\%, Figs. 1, 2, 3, 4, 5, 6, 7, 8, and 9), followed by the hip joint (6.3\%, Fig. 7) followed by the ankle joint (3.1\%, Fig. 2) with the right-sided predilection (65.6\%) more common than the left side (34.4\%) (Table 1).

All cases underwent arthroscopy and the final diagnosis was synovial chondromatosis in 14 patients $(43.8 \%$, Figs. 2, 3, and 4), PVNS in ten patients (31.2\%, Figs. 5 and 6), and lipoma arborescens in five patients $(15.6 \%$, Fig. 1). Synovial chondrosarcoma (Fig. 8), synovial hematoma (Fig. 7), and synovial ganglion cyst (Fig. 9) each in one patient (3.1\%), (Table 2). Regarding the sex predilection, all of these groups occur more in males compared to females.

Regarding synovial chondromatosis, MRI was unable to diagnose the malignant transformation in one case, 

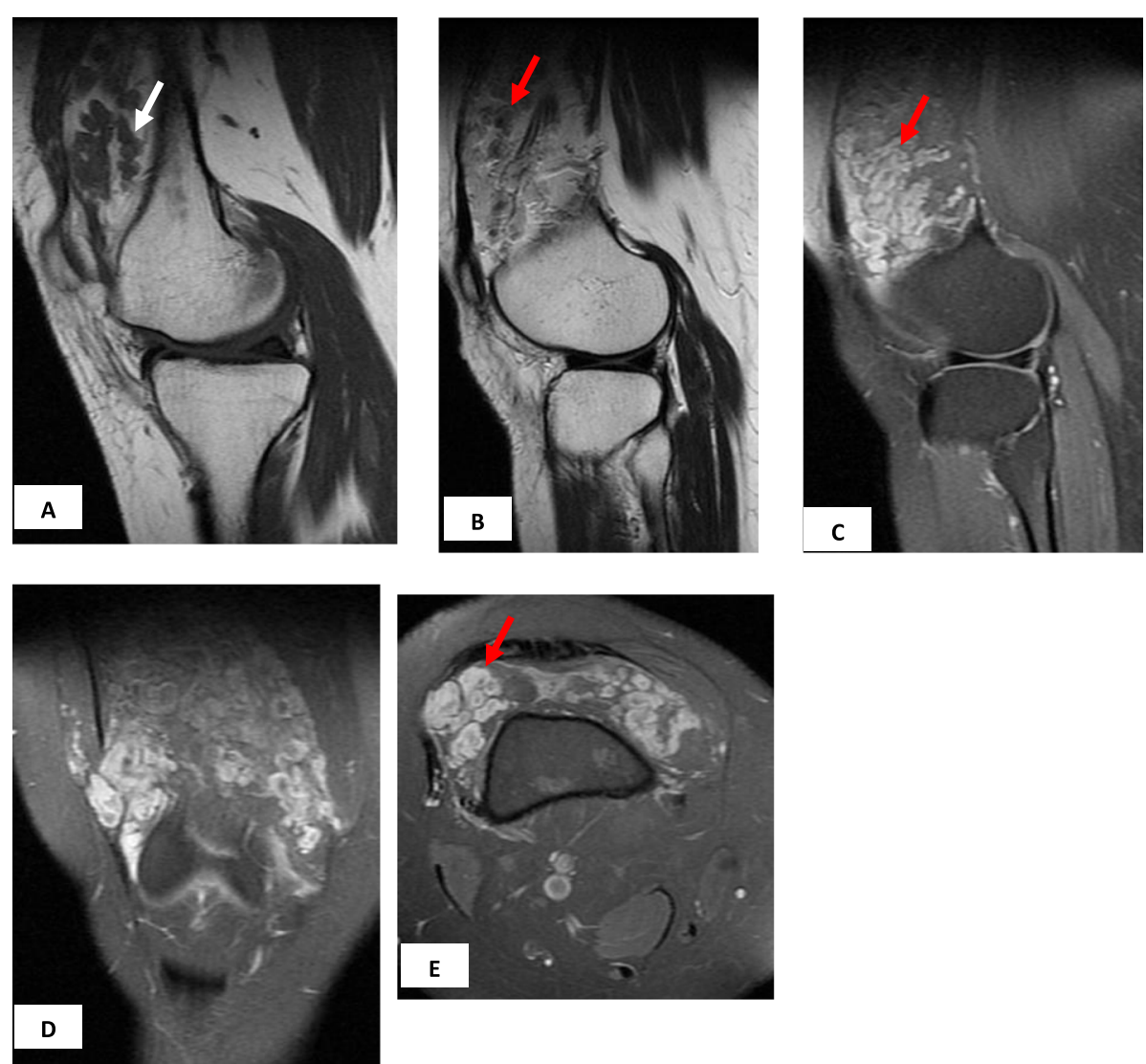

Fig. 4 Right knee synovial chondromatosis in 48-year-old male. Sagittal T1 (a), sagittal T2 (b), sagittal STIR (c), coronal STIR (d), and axial STIR (e) show non-mineralized equal size nodules showing low signal at T1 (white arrow), high at T2 and STIR (red arrows)

revealed by arthroscopy to be synovial chondrosarcoma (Table 2, Fig. 8).

The concordance of MRI in relation to the gold standard was $96.6 \%$ accuracy, $91.7 \%$ sensitivity, 99\% specificity, 52.3\% PPV, and 99.9\% NPV (Table 2).

The inter-observer agreement for evaluating the intraarticular synovial lesions using weighted kappa statistic was 0.83 indicating excellent agreement.

\section{Discussion}

Many of the diseases causing intra-articular masses have characteristic MRI imaging findings that enables a confident diagnosis making the differential diagnosis for such intra-articular masses somewhat limited [2].

In the current study, males were affected more than females and the knee joint was the most affected joint in agreement with Patrick J. Sheldon et al. [2] and Daniel Peixoto et al. [7] who reported similar sex and site incidence.

Synovial chondromatosis is a benign monoarticular disorder of uncertain etiology; more common in male and also more common in the knee joint, usually seen in the third to fifth decades. The disease is characterized by synovial proliferation and metaplastic transformation together with multiple formations of cartilaginous nodules. In the initial active phase of the disease in which there is synovial proliferation and formation of cartilaginous nodules intra synovial while in the final inactive phase of the disease, there are persistent nodules that may breakaway into the joint space. About $25-30 \%$ of the patients do not show calcification and the treatment is synovectomy with about 25\% recurrence rate. Associated marginal erosions, late secondary degenerative joint disease, and small joint effusion may be associated with synovial chondromatosis $[2,6-11]$.

Synovial chondromatosis can be classified into two types; primary type in which there is multiple intraarticular uniform-sized loose bodies which often calcify in its peripheral and central parts, and secondary type in which there is injury to the hyaline cartilage related to trauma, osteoarthritis, infectious 

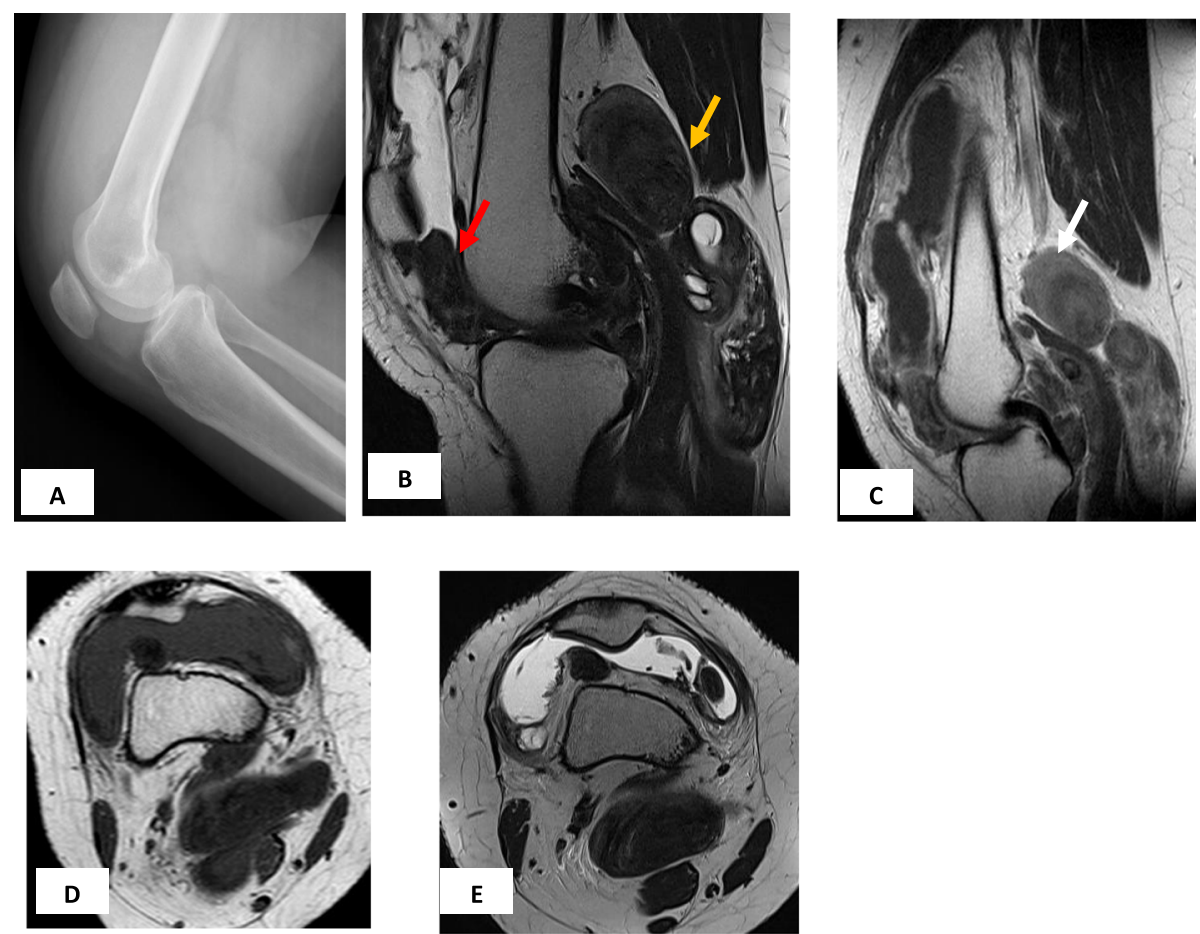

Fig. 5 Right knee diffuse PVNS in 42-year-old female. Lat X-ray (a) unremarkable, sagittal T2 (b), Sag T1 post-contrast (c), axial T1 (d), and axial T2 (e) show lobulated, mass-like (red arrow) heterogeneous enhanced (yellow arrow) synovial proliferation with characteristic low signal intensity, no articular erosions with large popliteal extra-articular component (white arrow). Sagittal T2 demonstrate massive synovial proliferation with low signal foci of hemosiderin deposition (red arrow)

arthritis, or osteochondritis dissecans and characterized by the presence of different shapes and sizes intra-articular loose bodies with various ring calcifications $[10,12,13]$.

In the current study; synovial chondromatosis was found in 14 patients (43.8\%) and their MRI appearance was variable depending on the proportion of proliferative synovium and formation of calcified nodules. Non-mineralized lesions show low signal at T1WI and high signal at T2 and STIR while calcified lesions display low signal at T1, low signal intensity of the periphery of the nodules, and high signal of the central bone marrow fat on T2WI in agreement with Patrick J. Sheldon et al. [2], Daniel Peixoto et al. [10], and Jie Wen et al. [13] who reported similar imaging features.

Synovial chondrosarcoma is remarkably uncommon and unclear whether it originates from malignant degeneration of synovial chondromatosis or de novo making the differentiation between them radiologically hard. Conventional radiographs usually show intraarticular soft-tissue mass that may contain calcified bodies. Both synovial chondrosarcoma and synovial chondromatosis may show bone erosions $[2,11]$.
Scott Evans et al. [11] reported that the true incidence of synovial chondrosarcoma is unknown and in their study included 800 patients and reported that $0.6 \%$ of chondrosarcomas were originating on top of primary synovial chondromatosis. They postulated that both conditions have similar patterns of clinical presentation with joint swelling, pain, and joint restricted movement. Many authors mentioned that found clinical and radiological criteria are unable to differentiate between both of them as there is usually noteworthy overlap with no definitive imaging features to separate both $[11,14,15]$.

We encountered in the current study one patient whose MRI showed thickened low signal synovium at $\mathrm{T} 1$, high at $\mathrm{T} 2$ with multiple low signal calcified bodies and initially diagnosed as synovial chondromatosis, but arthroscopy later revealed synovial chondrosarcoma in agreement with Patrick J. Sheldon et al. [2] and Scott Evans et al. [11] who mentioned that the imaging differentiation of synovial chondrosarcoma from synovial osteochondromatosis is difficult.

Scott Evans et al. [11] concluded that multiple recurrences and the development of bone marrow invasion are highly suspicious of osteochondromatosis malignant transformation. 

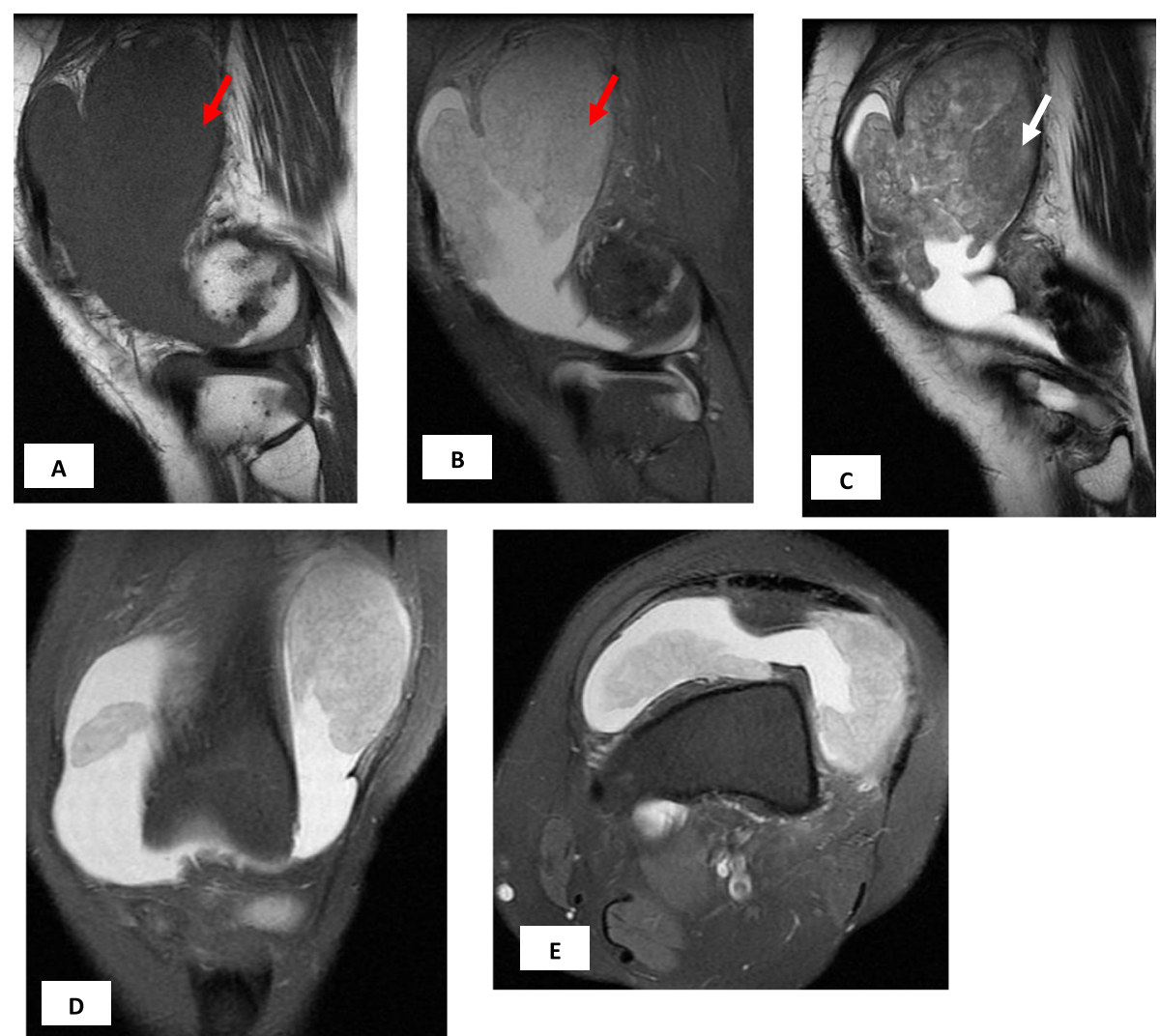

Fig. 6 Right knee diffuse PVNS in 40-year-old male. Sagittal T1 (a), sagittal STIR (b), sagittal T2 (c), coronal STIR (d), and axial STIR (e) show large lobulated, mass-like synovial proliferation with low signal intensity (red arrow), no articular erosions. Sagittal T2 demonstrate massive synovial proliferation with low signal foci of hemosiderin (white arrow) and associated joint effusion

PVNS is a proliferative synovial benign disorder that may affect the joints, tendon sheaths, or bursae and usually occurs in young to middle aged adults and extremely rare to be polyarticular. It can be diffuse intra-articular or less commonly focal and the knee is the most frequently affected joint with $50 \%$ recurrence rate after synovectomy. The affected joint by PVNS may appear normal or may show periarticular soft-tissue swelling in X-ray. Bone mineralization and joint spaces are specifically preserved until late in the disease [2].

MRI was able to diagnose all cases of PVNS in the current study, all of which was found in the knee joint with male predominance in agreement with Patricket al [2]. who mentioned similar site and sex incidence.

In the current series, all cases of PVNS were diffuse and MRI showed lobulated margin mass-like proliferative synovium with low signal foci at all pulse sequences due to deposition of hemosiderin in agreement with Patricket al [2]., A. Rodríguez Pan et al. [3], and AynurTuran et al. [4], who reported that the diffuse form
PVNS is the commonest form and reported similar imaging appearance.

MRI reveals prominent diffuse villous or nodular proliferation of synovium and associated joint effusion. Synovial thickening is visualized as an intermediate to low signal intensity on T1WI. There are low signal intensity areas due to the hemosiderin on T2WI. Particularly, hemosiderin appears as blooming artifacts on gradient echo (GRE) images because of its magnetic susceptibility, which is almost pathognomonic in all forms of PVNS. Lesions may show evident contrast enhancement after administration of IV gadolinium denoting the synovial proliferation high vascularity $[3,4,16,17]$.

Lipoma arborescens is an intra-articular uncommon lesion, usually monoarticular and seen often in the knee. In lipoma arborescens, there is villous synovial proliferation resulting from substitution of the subsynovial tissue by mature fat cells. The exact reason is unidentified, but the most reasonable hypothesis suggests a nonspecific synovial response to traumatic or inflammatory stimuli more willingly 

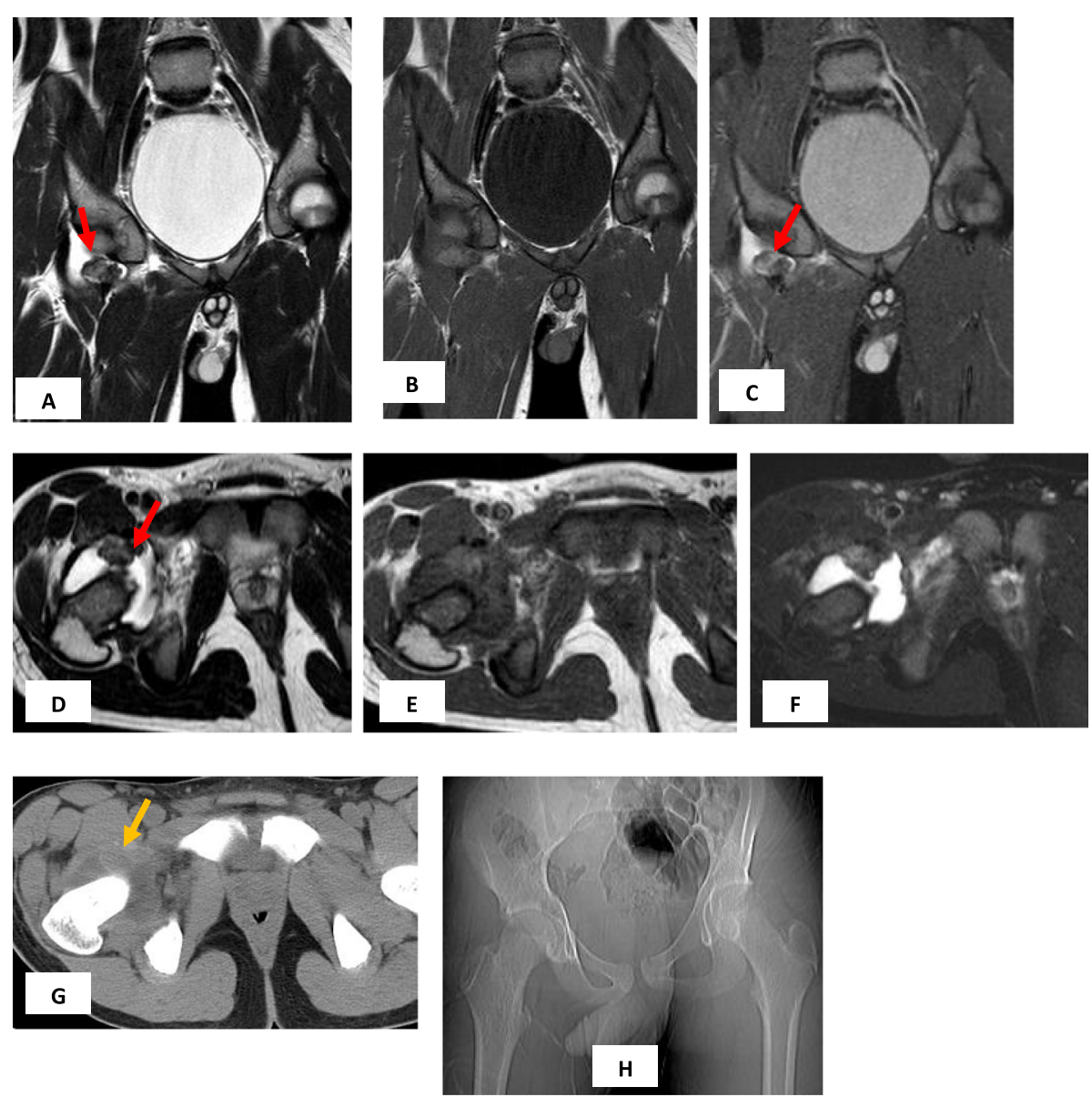

Fig. 7 Right hip post traumatic synovial hematoma in 25-year-old male. Coronal T2 (a), T1WI (b) and STIR (c), axial T2 (d), T1 (e), and STIR (f) show low signal mass-like synovial lesion (red arrows) with low signal intensity of hemosiderin and associated joint effusion. Axial CT (g) shows high density hematoma (yellow arrow). X-ray of the pelvis (h) unremarkable

than a neoplasm. Lipoma arborescens can be confidently diagnosed because of its characteristic imaging findings $[1,2,18-24]$.

MRI was able to diagnose all the cases of lipoma arborescens in the current series, all complained of painless swelling of the knee and they appear in MRI as intra-articular frond like fat-containing masses at the supra-patellar region (high signal at T1, T2, and suppressed at STIR) and was proved by arthroscopy in agreement with Pushpender Gupta et al. [18] who reported similar clinical presentation, and reported its more common predilection to the supra-patellar recess of the knee. $\mathrm{He}$ also mentioned that the MRI findings of intraarticular frond like fat-containing masses with added joint effusion are characteristic feature for lipoma arborescens.

We encountered one case (3.1\%) with synovial ganglion cyst in the current study in agreement with Sayaka
Kodaira1 et al. [25] and Partha Saha et al. [26] who reported similar rare incidence.

Sayaka Kodaira1 et al. [25] reported that intraarticular ganglion cysts are frequently seen in the dorsal wrist, shoulder, and palm but rarely to be intra-articular in the knee joint, usually detected as incidental MRI findings. Knee ganglion cysts may arise from both menisci, cruciate ligaments, subchondral bone, or popliteal tendon. The differential diagnosis of it may include synovial chondromatosis, PVNS, synovial hemangioma, and meniscal or parameniscal cysts.

Intra-articular ganglion cysts are frequently seen in the dorsal wrist, shoulder, and palm but rarely to be intraarticular in the knee joint, usually detected as incidental MRI findings $[1,2,7,27]$.

In our study, the ganglion cyst was located at the infrapetallar region (Hofa's fat) near the meniscus displaying low signal at T1, mixed low and high 

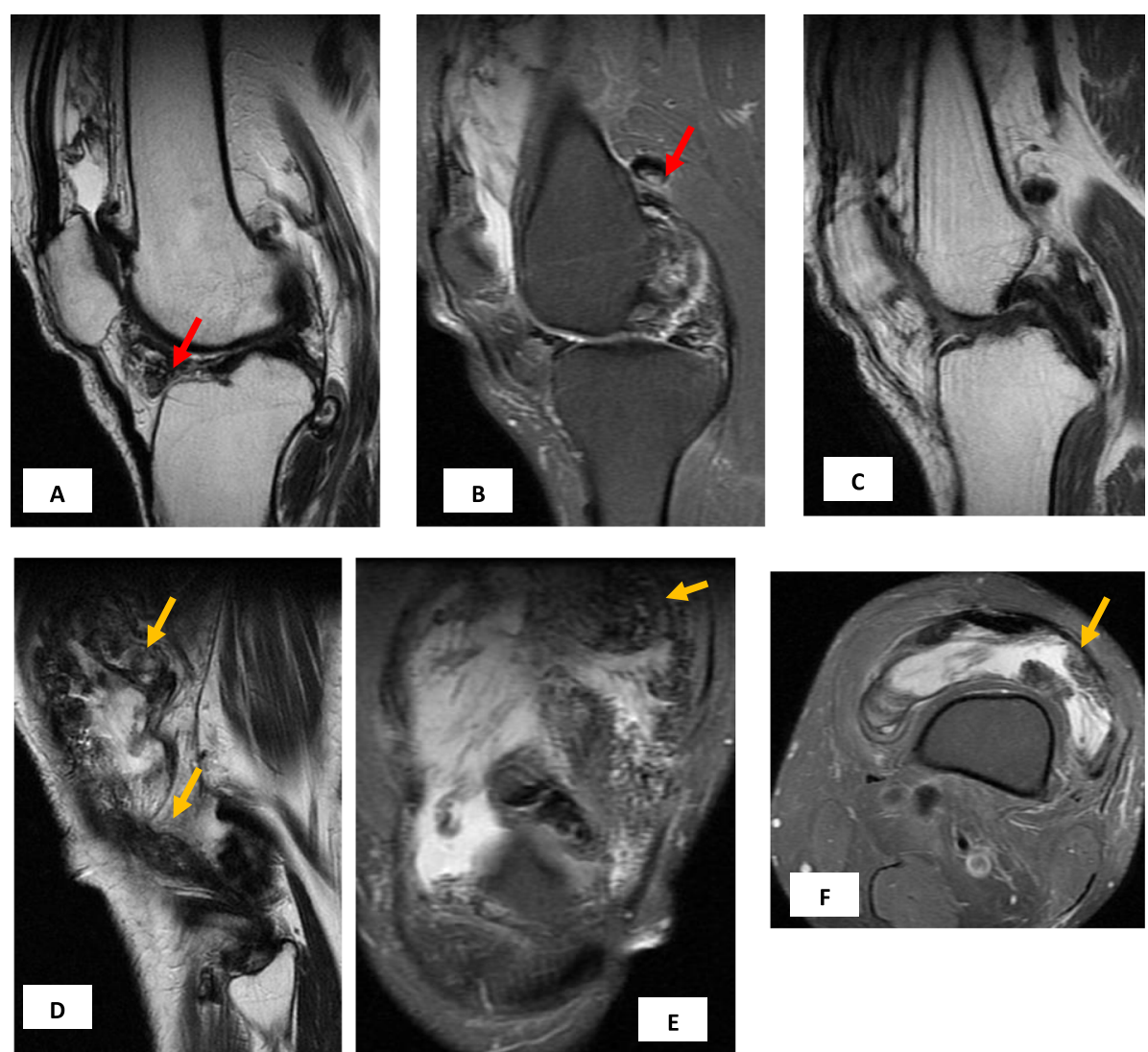

Fig. 8 Right knee synovial chondrosarcoma in 58-year-old male. Sagittal T2 (a, d), sagittal STIR (b), sagittal T1 (c), coronal STIR (e), and axial STIR (f) show unequal size low signal nodules at all series (red arrow) with synovial heterogeneous thickening (yellow arrows), MRI shows the possibility of synovial chondromatosis but arthroscopy reveals synovial chondrosarcoma

signal at T2 and STIR, and was confirmed by arthroscopic removal of the lesion in agreement with [26].

Synovial hematoma was observed in one patient (3.1\%) of the study group patients with history of trauma; CT in this patient reveals no fracture lines and US reveals effusion; MRI reveals mass-like lesion within the effusion displaying low signal hematoma with blooming effect at T2 in agreement with Vishal Kumar Jain et al. [28] who mentioned that in intraarticular hemorrhage regardless the cause, ironcontaining hemosiderin is kept in synovial tissue forming proliferative reaction and rusty discoloration. This hemosiderin causes typical T2WI blooming along the synovium, as seen in our patient. This blooming due to synovial hemorrhage can occur in many instances as in hereditary clotting factor deficiency diseases (e.g., hemophilia), trauma, use of anticoagulant, psoriatic or rheumatoid arthritis, collagen diseases, osteoarthritis, hemochromatosis, PVNS, sickle cell anemia, scurvy, synovial hemangioma, and myeloproliferative diseases.
The concordance of MRI in relation to the gold standard was $96.6 \%$ accuracy, $91.7 \%$ sensitivity, 99\% specificity, $52.3 \%$ PPV, and $99.9 \%$ NPV in agreement with Patrick et al. [2] and Scott Evans et al. [11] who mentioned difficulty for MRI to predict malignant transformation of synovial chondromatosis.

Using weighted kappa statistic, the inter-observer agreement for evaluating the intra-articular synovial lesions was 0.86 denoting excellent agreement in agreement with [6].

Limitation of the study is small number of patients that hinders us from studying other synovial intra-articular lesions during the study duration; large multicenter study is of value for better understanding of other varieties not encountered in our study.

\section{Conclusion}

In conclusion, good correlation was observed between MRI and arthroscopy in diagnosis of intra-articular synovial masses and mass-like lesions. 

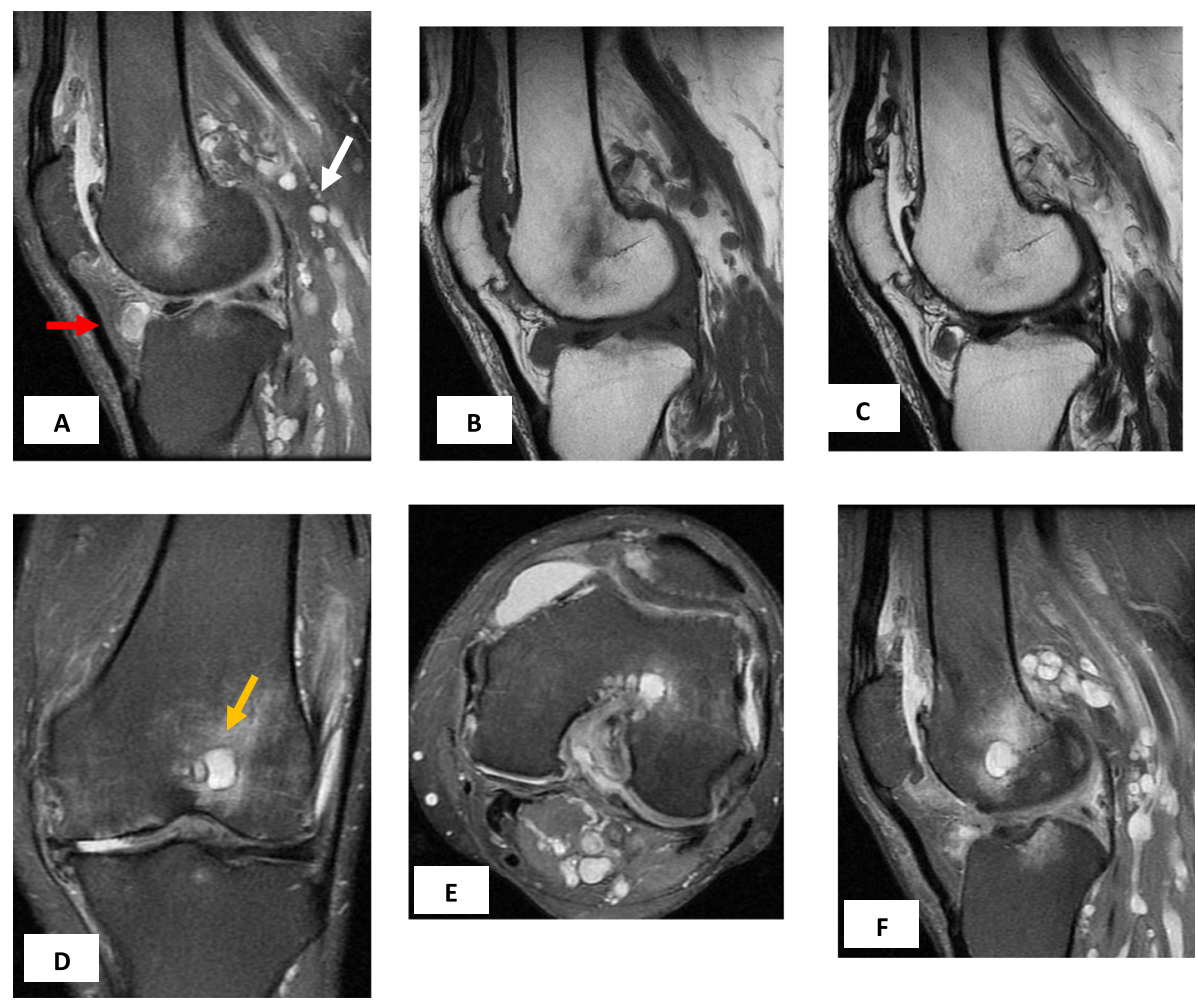

Fig. 9 Right knee synovial ganglion cyst at Hofa's fat in 61-year-old male. Sagittal STIR (a, f), sagittal T1 (b), sagittal T2 (c), coronal STIR (d), and axial STIR (e) show marked osteoarthritis, lower femur cyst (Geod, yellow arrow), popliteal varicose veins (white arrow), and synovial ganglion cyst (red arrow) displaying low signal at T1 and T2, mixed low and high at STIR

Table 1 distribution of the patients regarding sex, joint affection, and laterality

\begin{tabular}{lll}
\hline & Number & Percent \\
\hline Sex & 20 & $62.5 \%$ \\
Male & 12 & $37.5 \%$ \\
Female & & \\
Joint affected & 29 & $90.6 \%$ \\
Knee joint & 2 & $6.3 \%$ \\
Hip joint & 1 & $3.1 \%$ \\
Ankle joint & & \\
Joint laterality & 21 & $65.6 \%$ \\
Right side & 11 & $34.4 \%$ \\
Left side & & \\
\hline
\end{tabular}

Table 2 MRI diagnosis of the synovial lesions compared to arthroscope and their concordance

\begin{tabular}{llll}
\hline & MRI diagnosis & Arthroscopy & Total \\
\hline 1. Synovial chondromatosis & \multicolumn{1}{c}{15} & 14 & $14(43.8 \%)$ \\
2. PVNS & 10 & 10 & $10(31.3 \%)$ \\
3. Lipoma arborescence & 5 & 5 & $5(15.6 \%)$ \\
4. Synovial chondrosarcoma & - & 1 & $1(3.1 \%)$ \\
5.Synovial hematoma & 1 & 1 & $1(3.1 \%)$ \\
6. Ganglion cyst & 1 & 1 & $1(3.1 \%)$ \\
Total & 32 & 32 & 32 \\
Concordance of MRI and arthroscopy & & \\
$\quad$ Accuracy & $96.9 \%$ & & \\
Sensitivity & $91.7 \%$ & & \\
Specificity & $99 \%$ & & \\
PPV & $52.3 \%$ & & \\
NPV & $99.9 \%$ & & \\
\hline
\end{tabular}




\section{Acknowledgments}

No acknowledgments.

\section{Authors' contributions}

NM collected patient data, design of the study and performed the statistical analysis, and is responsible for correspondence to journal. TH wrote the manuscript, image processing, and collection of patient's images. All authors have read and approved the manuscript.

\section{Funding}

No finding sources.

\section{Availability of data and materials}

The datasets used and analyzed during the current study are available from the corresponding author on reasonable request.

\section{Ethics approval and consent to participate}

The study was approved by the ethical committee of Faculty of Medicine, Zagazig University and an informed written consent was taken from all subjects. The date of the approval of the protocol was February 2018 form the department of radiology, faculty of medicine, Zagazig university. The committee has no reference number only the date.

\section{Consent for publication}

All patients included in this research gave written informed consent to publish the data contained within this study. If the patient was less than 16 years old, deceased, or unconscious when consent for publication was requested, written informed consent for the publication of this data was given by their parent or legal guardian.

\section{Competing interests}

The authors declare that they have no competing interests.

Received: 5 August 2019 Accepted: 13 January 2020

Published online: 21 January 2020

\section{References}

1. Vanhoenacker FM, Van Goethem JWM, Vandevenne JE, Shahabpour M (2001) Synovial tumors. In: De Schepper AM, Parizel PM, De Beuckeleer L, Vanhoenacker $F$ (eds) Imaging of soft tissue tumors, vol 19. Springer, Berlin, pp 311-324

2. Sheldon PJ, Forrester DM, Learch TJ (2005) Imaging of intraarticular masses. RadioGraphics 25:105-119

3. Rodríguez Pan A, Martínez Calvo A, Ríos Reboredo A, Méndez Díaz C, Soler Fernández R (2012) Articular and periarticular tumors: differential diagnosis using magnetic resonance imaging. Radiología. 54(1):21-44

4. Turan A, Celtikçi P, Tufan A, Öztürk MA (2017) Basic radiological assessment of synovial diseases: a pictorial essay. Eur J Rheumatol 4:166-174

5. Dash KK, Gavai PV, Wade R, Rajani A (2016) It's not what it looks like: challenges in diagnosis of synovial lesions of the knee joint. J Exp Orthop 3:5

6. Fleiss $J \mathrm{~L}$ (1981) Statistical methods for rates and proportions, 2nd edn. Wiley, New York, pp 212-255

7. Peixotoa D, Gomesb M, Torresb A, Miranda A (2018) Arthroscopic treatment of synovial chondromatosis of the ankle. Rev Bras Ortop 53(5):622-625

8. Brodsky JW, Jung KS, Tenenbaum S (2013) Primary synovial chondromatosis of the subtalar joint presenting as ankle instability. Foot Ankle Int 34(10): 1447-1450

9. Doral MN, Uzumcugil A, Bozkurt M, Atay OA, Cil A, Leblebicioglu G et al (2007) Arthroscopic treatment of synovial chondromatosis of the ankle. J Foot Ankle Surg 46(3):192-195

10. Liu X, Wan S, Shen P, Qiu Y, Sah MK et al (2019) Diagnostic accuracy of synovial chondromatosis of the temporomandibular joint on magnetic resonance imaging. PLoS ONE 14(1):e0209739. https://doi.org/10.1371/ journal.pone.0209739ID

11. Evans S, Boffano M, Chaudhry S, Jeys L, Grimer R (2014) Synovial chondrosarcoma arising in synovial chondromatosis. Sarcoma. https://doi. org/10.1155/2014/647939 Hindawi Publishing Corporation, Article ID 647939, 4 pages

12. Teixidor Viñas M, Martinez de la Haza D, Santos Gomez I, RojoSanchis N, Santo Panero P, et al. Synovial knee disease: MRI differential diagnosis. ES,
Hospitalet de Llobregat/ES, Sant Boi de Llobregat/ES,Barcelona/E. 2013. DOI: https://doi.org/10.1594/ecr2013/C-2443

13. Wen J, Liu H, Xiao S, Li X, Fang K, Tang Z, Cao S et al (2018) Synovial chondromatosis of the hip joint in childhood, a case report and literature review. Medicine 97(51):e13199

14. Murphy MD, Vidal JA, Famburg-Smith JC, Gajenski DA (2007) Imaging of synovial chondromatosis with radiologic-pathologic correlation. Radiographics 27:1465-1468

15. Zamora EE, Mansor A, Vanel D et al (2009) Synovial chondrosarcoma: a report of two cases and literature review. Eur J Radiol 72(1):38-43

16. Narváez JA, Narváez J, Ortega R, De Lama E, Roca Y, Vidal N (2003) Hypointense synovial lesions on T2- weighted images: differential diagnosis with pathologic correlation. AJR Am J Roentgenol 181:761-769

17. Riis RGC (2018) Assessing synovitis with conventional static and dynamic contrast-enhanced magnetic resonance imaging in knee osteoarthritis. Dan Med J 65(4):B5464

18. Gupta P, Potti TA, Wuertzer SD, Lenchik L, Pacholke DA (2016) Spectrum of fat-containing soft-tissue masses at MR imaging: the common, the uncommon, the characteristic, and the sometimes confusing. RadioGraphics 36:753-766

19. Kamaci S, Doral MN, Ergen FB, Yucekul A, Cil A (2015) Lipoma arborescens of the knee. Knee Surg Sports Traumatol Arthrosc 23(8):2196-2201

20. Sanamandra SK, Ong KO (2014) Lipoma arborescens. Singap Med J 55(1):510 quiz 11

21. Natera L, Gelber PE, Erquicia JI, Monllau JC (2015) Primary lipoma arborescens of the knee may involve the development of early osteoarthritis if prompt synovectomy is not performed. J OrthopTraumatol 16(1):47-53

22. Bejia I, Younes M, Moussa A, Said M, Touzi M, Bergaoui N (2005) Lipoma arborescens affecting multiple joints. Skelet Radiol 34(9):536-538

23. Howe BM, Wenger DE (2013) Lipoma arborescens: comparison of typical and atypical disease presentations. Clin Radiol 68(12):1220-1226

24. Dogramaci Y, Kalaci A, Sevinç TT, Atik E, Esen E, Yanat AN (2009) Lipoma arborescens of the peroneus longus and peroneus brevis tendon sheath: case report. J Am Podiatr Med Assoc 99(2):153-156

25. Kodaira S, Nakajima T, Takahashi R, Moriya S et al (2016) A case of intraarticular ganglion cysts of the knee joint: correlation between arthroscopic and magnetic resonance imaging. BMC Med Imaging 16:36

26. Saha P, Bandyopadhyay U, Mukhopadhyay AS, Kundu S, Mandal S (2015) Ganglion cyst of knee from Hoffa's fat pad protruding anterolaterally through retinacular rent: a case report. J Orthop Case Rep 5(3):69-71

27. Caracciolo JT, Henderson-Jackson E, Binitie O (2019) Synovial sarcoma of bone: sarcoma typically of soft tissues presenting as a primary bone tumor. Radiol Case Rep 14:204-207

28. Jain VK, Singh RK, Kumar S, Netam SS, Jain SG, Shah PJ (2016) Hemosiderotic synovitis: highlighting the role of $\mathrm{T}^{*}$ weighted sequence in skeletal MRI. Egypt J Radiol Nucl Med 47:1511-1513

\section{Publisher's Note}

Springer Nature remains neutral with regard to jurisdictional claims in published maps and institutional affiliations.

\section{Submit your manuscript to a SpringerOpen ${ }^{\odot}$ journal and benefit from:}

- Convenient online submission

- Rigorous peer review

- Open access: articles freely available online

- High visibility within the field

- Retaining the copyright to your article

Submit your next manuscript at $>$ springeropen.com 